\title{
AGENTES FARMACOLOGICOS QUE AFECTAN AL FETO
}

\author{
Di. Alberto Duarte-Contreras M.D.* \\ Dr. José G. Coronel-Becerra M.D.** \\ $D r$. Luis F. Luzardo M. M.D.***
}

Oímos a uno de nuestros maestros en alguna oportunidad la siguiente máxima: "no olvidemos que el Obstetra es el pediatra del feto" (1). Ello nos hizo reflexionar muy seriamente en los riesgos a que está expuesto el feto con el cúmulo inagotable de agentes farmacológicos que se administran a la madre, muchas veces de manera indiscriminada. Nos detendremos a estudiar únicamente aquellos factores que son de uso corriente en nuestro medio.

Está prácticamente demostrado que todo medicamento puede atravesar la "barrera placentaria" y alcanzar en el feto concentraciones inferiores o iguales a las del torrente circulatorio materno, y producir en él efectos tanto terapéuticos como tóxi$\cos y$ aún teratológicos (112).

Sabemos que la acción teratógena de ciertas drogas solo tiene importancia cuando se administran antes de la undécima semana de la gestación; y a su vez, ciertas drogas administradas después de la undécima semana pueden producir en el feto normalmente desarrollado, un efecto patológico y aún ser causantes de la muerte fetal (96).

El paso transplacentario de una droga se efectúa por diversos meca- nismos: transporte activo, ultrafijación, liposolubilidad, procesos especiales, ruptura de las vellosidades placentarias, cambios de permeabilidad por lesiones patológicas, y finalmente por difusión. El proceso de difusión es el de mayor importancia y en él las moléculas pasan de un área de mayor a una de menor concentración. La difusión se efectúa generalmente de manera directa; pero también puede hacerse de manera indirecta cuando la droga se metaboliza en el organismo materno y pasan luego al feto los metabolitos por el proceso de difusión (2), (85).

Todas las substancias en dilución acuosa tienen un paso transplacentario más fácil que las de dilución oleosa y así mismo el paso es más difícil entre mayor sea el peso molecular (por debajo de 600 todos los medicamentos pasan fácilmente).

Algunos estados patológicos como la diabetes, la toxemia, la eritroblastosis, trastornos circulatorios mater-

* Médico Jefe del Departamento Gíneco-Obstétrico del Hospital San Juan de Dios, de Cúcu, ta, Colombia.

* * Médico Jefe del Departamento de Pediatría del Hospital San Juan de Dios, de Cúcuta.

*** Médico Adjunto del Departamento Gíneco-Obstétrico del Hospital San Juan de Dios, de Cúcuta. 
nos, pueden debilitar o eliminar la barrera placentaria y favorecer así el paso de la droga a través de la placenta.

Los factores físicos o farmacológicos que actúan sobre la madre pueden tener sobre el feto una actividad nociva bien sea depresiva o teratológica de acuerdo con determinados factores que anunciaremos en seguida: (3), (23).

1. La edad del embarazo: es indispensable al administrar factores farmacológicos conocer tanto el momento embriológico como el curso del embarazo ya que los riesgos teratológicos los encontramos en el período de embriogénesis o sea en los tres primeros meses, en cambio los riesgos de enfermedad fetal los tendremos durante todo el embarazo pero de manera preferencial al final de éste y durante el trabajo de parto.

2. Cantidad de droga administrada a la madre y cantidad de droga que pasa al feto; pero la cantidad de droga administrada a la madre puede ser muy relativa en algunas oportunidades ya que dosis muy pequeñas pueden producir serias malformaciones (por ejemplo 100 miligramos de Talidomida pueden producir serias malformaciones fetales si se administra entre la cuarta y la octava semana).

3. Duración de la administración de la droga.

4. Degradación que sufra en el organismo materno y fetal: hay drogas que se concentran en los tejidos fetales al parecer por la incapacidad que éstos tienen para metabolizarlos por razón de su inmadurez.

5. Velocidad de eliminación en la madre.
6. Inmunidad materna para la droga transmitida al feto.

7. Sensibilidad fetal a la droga la que puede ser diferente a la sensibilidad materna.

8. Rapidez con que atraviesa la ba. rrera placentaria.

Sabemos que cualquier tratamiento a la madre es un verdadero tratamiento al feto, necesítelo éste o no, y exige por ende, una constante preocupación para evitar complicaciones fetales que pueden tener graves e irreparables consecuencias.

Es de lógica considerar que todas las drogas se administran a dosis terapéuticas, pero desafortunadamente no conocemos todos los factores físico-químicos o biológicos que condicionan el paso transplacentario ni tampoco conocemos a cabalidad la manera de acción de las drogas sobre el embrión (4).

\section{I - VACUNAS}

Las vacunas hacen aparecer en el suero materno anticuerpos transmisibles al feto, o refuerzan su título si ya existe, asegurando así la inmunidad pasiva del recién nacido, si bien es cierto que esta inmunidad no es ni completa ni duradera ya que el nivel de anticuerpos decrecerá progresivamente y con cierta rapidez en el primer mes de la vida en la mayoría de los casos.

Las vacunas con gérmenes muertos, las anatoxinas, y las vacunas con virus inactivos son bien soportadas por el feto, como la vacuna Salk y la vacuna antitetánica (4) (5).

Se ha discutido mucho el peligro de la vacunación antivariólica; Greenberg niega el efecto perjudicial contra el embrión aplicada en el primer trimestre (82) en cambio McArthur 
anota un aumento importante en la mortalidad fetal cuando se aplica en el segundo y en el tercer mes del embarazo (83). Pero ante la posibilidad de una embriopatía viral no se aconseja aplicarla durante los tres primeros meses de gestación, ni cuando haya antecedente de aborto repetido.

Se recomienda no administrar vacunas de gérmenes vivos durante el embarazo, de manera especial durante los tres primeros meses (vacuna contra viruela, polio, fiebre amarilla, sarampión, tuberculosis). Y cuando su administración se haga indispensable, se deberán tomar precauciones especiales como la administración a dosis fraccionadas (3).

\section{II - DROGAS DEL SISTEMA NERVIOSO}

\section{MORFINA}

Todos los narcóticos y anestésicos tienen una constante elevada de liposolubilidad, lo que les proporciona un rápido tránsito transplacentario con fácil e inmediato acceso al feto.

No se han descrito malformaciones en productos de madres morfinómanas pero sí se ha comprobado una elevada mortalidad perinatal, frecuentes abortos y aumento en el índice de prematurez, como también una baja considerable en el peso de los recién nacidos ( 2.600 grs. en promedio comparado con 3.400 grs. de los productos controles). (6).

De años atrás se conoce un síndrome en los recién nacidos de madres morfinómanas quienes reciben de 2 a 45 mgrs. diarios, síndrome que presenta caracteres muy semejantes a los que pudiera ocasionar en la madre la supresión de la droga: agitación, irritabilidad, Ilanto excesivo, insomnio, temblor, vómito, diarrea, bostezos, estornudos, dificultad res- piratoria, fiebre y aún convulsiones; puede presentarse ictericia: este síndrome aparece al segundo o tercer día de vida. El tratamiento se hace con substitución (7).

A toda embarazada drogadicta se le debe suspender la droga al séptimo mes y hacerle de inmediato una terapia de substitución (84) advirtiéndole que se puede presentar un exceso de movilidad fetal.

Cuando se administra morfina $u$ opiáceos durante el trabajo de parto producen en el recién nacido una depresión respiratoria severa, dada su acción directa depresora sobre el centro correspondiente, con cianosis, alteraciones pupilares y sintomas de "abstinencia", el pH es más lento en normalizarse, hay aumento del $\mathrm{CO} 2$ y baja de las bases tampones.

Al inyectar 15 mgrs. de Morfina a la madre por vía venosa dos o seis minutos antes de ligar el cordón umbilical, ésta se encontrará en la orina del recién nacido hasta cuatro días después del parto. Si se inyecta Meperidina (Demerol) 100 a 200 mgr. por vía venosa a la madre entre 40 minutos y $3 \frac{1}{2}$ horas antes de ligar el cordón umbilical, los niveles en la sangre fetal alcanzan un 45 a $106 \%$ de la concentración en la sangre materna (investigada simultáneamente) y aún se encuentra en la orina del recién nacido hasta 24 horas más tarde. Pero si el parto se efectúa antes de una hora o después de seis horas de haber administrado la Meperidina, los efectos narcóticos son mínimos (8).

Unos derivados de la Morfina como la N-ally-morfina (Nalorfina) administrados a la madre simultáneamente con la morfina o los opiáceos, pasan al recién nacido por la vena umbilical y ejercen una acción anti- 
depresiva muy notoria: su acción antagónica es mayor aún si se administran por vía umbilical (9) (10).

\section{ANESTESICOS POR INHALACION}

Todos los anestésicos empleados atraviesan la barrera placentaria y rápidamente se encuentra un equilibrio más o menos perfecto entre la concentración del producto químico en la sangre materna y en la sangre fetal. Cuando usamos éter, encontramos niveles muy iguales aún varios minutos después del parto. El ciclopropano da entre 58 y $94 \%$ del nivel materno. El Tricloretileno (Trilene) parece no acarrear peligros para el feto (88).

El 4-hidroxibutirato sódico $\circ \delta-\mathrm{OH}$, novedad en el campo de la anestesia obstétrica por su aparente inocuidad, puede producir hipoxia, depresión respiratoria y aún alteraciones en el equilibrio ácido básico del recién nacido durante la primera hora de vida por la acción directa sobre el centro respiratorio y por las modificaciones dinámicas sobre la contractilidad uterina (90) (89).

Todos los anestésicos generales deprimen al feto y pueden ser causa de anoxia fetal, a veces mortal. Su tratamiento consiste en someter a la madre a una suficiente oxigenación durante el tiempo de la administración del anestésico y luego oxigenar al recién nacido deprimido (113).

Se considera que el óxido nitroso es el que menos deprime y el ciclopropano el que más deprime (10), (12).

\section{BARBITURICOS}

Todos los barbitúricos producen sobre el feto una depresión del centro respiratorio acompañada de hipoxia y aún de anoxia. Los de ultracorta acción o tiobarbituratos atravie- san la placenta con gran rapidez dada su inmensa liposolubilidad y pueden detectarse en la sangre del cordón cuarenta y cinco segundos después de haber sido suministrados a la madre: gozan de escasa aceptación en obstetricia (12). Los de corta y los de larga acción cruzan fácilmente la barrera placentaria muy a pesar de su poca solubilidad y se les puede encontrar en el cordón umbilical en una concentración que alcanza el $71 \%$ del nivel materno en promedio, tres minutos después de haber sido administrados a la madre (11). Pero el efecto sobre el recién nacido es inferior si se compara con el efecto hipnótico profundo que ejerce sobre la madre, fenómeno que podemos explicar de la siguiente manera: si por ejemplo los centros maternos reciben una concentración de 50 mgrs. por litro, la barrera placentaria demora el paso hasta cuando el nivel materno haya caído a $10 \mathrm{mgr}$. por litro; además la circulación sanguínea de la vena umbilical pasa en parte por el hígado y hay también una dilución en la vena cava, siendo de esta manera menor aún la concentración de barbitúricos a nivel del sistema nervioso central del recién nacido.

La acción sobre el feto puede durar hasta 25 horas o más; sin embargo no se conocen lesiones permanentes que se les puedan atribuir.

Recordemos que el fenobarbital puede acentuar la hipoprotrombinemia del recién nacido cuando se administra a dosis altas y repetidas al final del embarazo (38). Puede tomar mayor actualidad este concepto por la utilización que se le está dando al Luminal para obtener reducción terapéutica de la bilirrubina indirecta (112), y reducción de bilirrubina total en hijos de mujeres trata- 
das con Luminal durante la gestación (113).

\section{RELAJANTES MUSCULARES}

Parece que no atraviesan la barrera placentaria; no se ha podido demostrar por ningún método la presencia del CURARE en la sangre del cordón umbilical (11).

EI SULFATO DE MAGNESIO de uso rutinario entre nosotros en el tratamiento de la eclampsia, puede, cuando lo empleamos a dosis altas y repetidas, disminuir la transmisión neuromuscular en la madre y en el recién nacido y producir depresión (86).

\section{TRANQUILIZANTES}

En general los tranquilizantes no son tóxicos para el feto, pero su empleo en las embarazadas a término, según la opinión de algunos autores, aumenta la ictericia neonatal (19) y según otros no producen variación alguna en la curva de bilirrubinas.

EI VALIUM y el LIBRIUM (derivados de la benzodiazepina) como también el MEPROBAMATO carecen de acción perjudicial sobre el feto y el recién nacido (90) (91).

La NIALAMIDA, del grupo ihdrazínico de fármacos inhibidores de la mono-amino-oxidasa, es de uso no aconsejado en obstetricia ya que pertenece a un grupo de fármacos de gran actividad y de acciones no bien conocidas ( 21 ).

EI HALOPERIDOL tan en boga hoy día, es considerado como inocuo por unos autores (21). Sin embargo, para otros produce con frecuencia reacciones extrapiramidales tales como parkinsonismo, distonía, diskinesia, crisis oculogíricas y akatisia (síntomas todos que cesan al suspender la dosis o al administrar agentes antiparkinsonianos tales como el Propanolol) y son enfáticos al aseverar que no se ha establecido el margen de seguridad de este medicamento durante el embarazo. Es una butirofenona que antagoniza el efecto estimulante de las anfetaminas, prolonga el sueño inducido por los barbitúricos y refuerza la acción de los depresores del sistema nervioso central, incluso los anestésicos generales 27 .

La CLORPROMAZINA usada a dosis repetidas hasta alcanzar totales de 500 a 600 mgrs. durante el trabajo de parto produce trastornos respiratorios en el feto $y$ en el recién nacido (20).

La RESERPINA se acumula con bastante rapidez en el feto y cuando se administra al final del embarazo puede originar en el recién nacido una angustia respiratoria caracterizada por congestión e hipersecreción óculonasal, retracción costal, bradicardia, somnolencia, letargia, anorexia y a veces hipotermia, síntomas que pueden durar hasta seis días. Budnik encontró este sindrome en el 16\% sobre 77 recién nacidos de madres tratadas con reserpina al final del embarazo (18). Desmonil observó cambios fisiológicos aún más profundos: recién nacidos con hipertonía, disminución de la actividad e hipotermia, todo antes de apreciarse la congestión nasal (92).

\section{TABACO}

Figura el tabaquismo como causa indiscutible de prematurez (13, 14), y muchos autores afirman categóricamente que los hijos de fumadoras son de tamaño reducido y bajo peso: opinan que mujeres fumadoras o que trabajan en la industria del tabaco son particularmente propensas a presentar abortos o mortinatos (15), 
(16), (17). La nicotina produce vasoconstricción, taquicardia, irritación de las mucosas, hiperacidez gástrica y pérdida de apetito. En el humo del tabaco hay un buen número de substancias consideradas venenos tisulares, tales como el monóxido de carbono, prefural, piridina, priolina, arsénico, níquel, óxido de carbono y otros más; y todas ellas se absorben por las mucosas bucal y faríngea aún sin aspiración profunda y pasan rápidamente al torrente circulatorio afectando directamnete al feto. El hábito de fumar ocasiona un aumento de la tasa de carboxihemoglobina tanto en la sangre materna como fetal; además la vasoconstricción producida por la nicotina afecta directamente al organismo fetal y disminuye el débito sanguíneo placentario, y la perturbación del apetito acarrea hipoalimentación materna. Recordemos que el organismo femenino tiene una gran sensibilidad a los efectos nocivos del tabaco sobre el sistema vascular (2).

\section{III - MISCELANEA}

HEXAMETONIO. Es un compuesto del amonio cuaternario, bloqueador ganglionar. Cruza la barrera placentaria y se concentra en el líquido amniótico (93). De la misma manera que puede producir íleus paralítico en la madre, lo ocasiona en productos prematuros nacidos de estas madres (94). El estreñimiento intenso que puede producir se alivia con prostigmine o con piridostigmina; en caso de que no ceda se suspenderá la droga.

MECLIZINE (Bonadoxina). La escuela sueca ha insistido en la posibilidad de efectos teratógenos dando una incidencia hasta del $12 \%$ cuando se prescribe entre la quinta y la sexta semanas; y han comprobado que en grandes dosis suministradas a ra- tas, es un potente agente teratogénico (95). Otros niegan su teratogenicidad en el ser humano (46) (45). Sin embargo la Oficina de Administración de Alimentos y Fármacos de Estados Unidos (U.S. Food and Drug Administration) el 5 de marzo de 1966 ordena que la Meclizina debe llevar una etiqueta que destaque el "peligro de deformidades congénitas si el fármaco es ingerido por embarazadas" (48). La doctora Apgar dice que aunque es poco probable que estas substancias de uso corriente sean teratógenas para el ser humano, no hay datos que comprueben 0 descarten la hipótesis (48).

FENACETINA. Puede causar anemia, metahemoglobinemia y sulfhemoglobinemia. Cuando la madre la toma por tiempo muy prolongado puede producir lesiones renales tanto en ella como en el feto (10).

SALICILATOS. Algunos autores sostienen que pueden producir un aumento de las manifestaciones hemorrágicas en el recién nacido de madres que han recibido fuertes dosis (24), (87), mas no se han observado las manifestaciones descritas en animales de experimentación.

QUININA. Usada a dosis terapéutica para el tratamiento del paludismo puede producir sordera a la embarazada, y autores como Nelson sostienen que puede ocasionar sordena congénita. Administrada a dosis alta y sostenida en cualquier edad del embarazo puede acarrear púrpura trombocitopénica tanto en la madre como en el feto (26). Sugieren que la trombocitopenia neonatal seguida a la administración de quinina a la madre es resultante del paso transplacentario de anticuerpos y quinina. Otros autores sostienen haber encontrado graves malformaciones fetales tales como aperturas septales inter- 
ventriculares, riñones poliquísticos, polisindactilia y hasta amelia total en productos de madres que ingirieron altas dosis en las primeras semanas del embarazo con el fin de provocar el aborto (28).

PIRIMETAMINA O Daraprin, es un antimalárico, potente antagonista, reductor o inhibidor del ácido fólico y de la reductasa del ácido fólico a punto tal que puede producir anemia megaloblástica y depresión de la medula ósea con trombocitopenia y leucopenia, a más de efectos teratogénicos como antifólico que es (98), (99). Es droga muy socorrida en el tratamiento de la Toxoplasmosis. Puede producir abortos si se la administra durante el primer trimestre del embarazo.

GRISEOFULVINA (Grisovin): la casa productora recomienda abstenerse de su uso bien sea oral o tópico clurante los tres primreos meses del embarazo por sus posibles efectos teratogénicos, pues en el campo experimental altera ciertos tejidos de crecimiento rápido tales como el epitelio de los tubos seminíferos (68).

EMETINA. No hay datos aceptables en relación con efectos teratogénicos del fármaco; sin embargo se aconseja cvitar su empleo durante el embarazo, a menos que su uso sea esencial (100).

CLORHIDRATO DE QUINACRINA O Atebrina: droga específica para el tratamiento de la giardiasis; se contraindica en el primer trimestre del embarazo por su posible teratogenicidad (100).

\section{IV - ANTIBIOTICOS Y SULFAS}

Los diferentes antibióticos atraviesan la barrera placentaria pero su paso se considera difícil y el nivel sanguíneo en la sangre fetal sólo alcan- za de un 25 a un $75 \%$ del de la madre. Está perfectamente demostrado y aceptado que no producen aborto. El paso es rápido para la cicloserina y la espiramicina; un poco menos rápido para la penicilina, la estreptomicina, la tetraciclina y el cloranfenicol; y más difícil aún para la clortetraciclina y la eritromicina. El paso también es más fácil en las primíparas que en las multíparas (3).

PENICILINA. Sesenta minutos después de la aplicación de una dosis de Penicilina se encuentran concentracoines en la sangre fetal hasta del $50 \%$, y también en concentración de utilidad terapéutica en el líquido amniótico: ello hace que se la emplee tanto en la profilaxis como en el tratameinto de las infecciones debidas a la ruptura de membranas (29). Pero muchos autores afirman que la prevención de las infecciones por vía transplacentaria es muy dudosa. No se conocen lesiones producidas por la penicilina en el recién nacido, aún con dosis altas, pero sí se han reportado casos de muerte fetal consecutivas a la reacción anafiláctica materna al administrar penicilina a la madre (reacción de Herxheimer).

ESTREPTOMICINA. Chassagne describe cuatro casos de lesiones del octavo par (sordera) atribuíbles a la administración de estreptomicina a la madre al comienzo del embarazo (30), (117). La estreptomicina apenas alcanza en el feto el $50 \%$ del nivel materno. Suministrada a la madre a dosis altas en el momento del parto puede producir síntomas de intoxicación en el recién nacido caracterizados por un coma prolongado que aparece 30 minutos después de administrada la droga y puede durar de 6 a 8 horas, y en ocasiones llevar a la muerte cuando la dosis administrada alcanza en el feto concentraciones de 0,5 a $1 \mathrm{gr}$. 
CLORANFENICOL. No se conocen casos de toxicidad en recién nacidos tras su uso en la madre, no así cuando se administra por el cordón umbilical directamente al feto, pudiendo producir en este caso el "síndrome gris" (102).

NOVOBIOSIN (Albamycin). Puede inhibir los procesos enzimáticos que se relacionan con el metabolismo de la bilirrubina; puede ser el causante de hiperbilirrubinemia en el recién nacido posiblemente por su afinidad al sistema glucoronil transferasa; esta afinidad crearía una insuficiencia relativa de la transferasa (35). Pero algunos autores creen que el uso de esta droga no produce retención del pigmento en el feto ni efecto alguno nocivo (31).

TETRACICLINA. Es aceptado hoy día que la tetraciclina administrada a la madre al final del embarazo se deposita en los huesos del feto bajo la forma de un complejo fluorescente; este depósito óseo de tetraciclina disminuye la velocidad de crecimiento del hueso, lo que se puede demostrar muy bien en el peroné mediante el método radiológico de Silverman y Day. También se puede encontrar más tarde fluorescencia positiva en los dientes y aún hipoplasia del esmalte o enamel si la tetraciclina se administra hacia la segunda mitad del cuarto mes del embarazo, época en que comienza la calcificación de los deintes. A la edad de 4 a 5 años los dos tercios incisales de la corona aparecen grisáceos con pigmentación amarillo oscura o café en el tercio cervical; y en los dientes severamente comprometidos puede haber cambios de forma (32), (33), (34), (118), (119). Parece que la clortetraciclina contenga menores efectos perjudiciales en el feto debido a su difícil paso transplacentario, como que apenas si alcanza a tener niveles del $25 \%$ de los maternos.

La DOXICICLINA (Vibramicina) presenta todos los defectos adversos comunes a la tetraciclina. Las reacciones de fotosensibilización también han sido observadas con bastante frecuencia después de la administración de la doxiciclina (42).

FURADANTINAS. Empleadas con excelente resultado en el tratamiento del seminoma (tumor de células germinales muy diferenciadas) impidiendo el crecimiento de las células germinativas. Nos preguntamos: no tendrán un efecto semejante sobre el embrión durante los tres primeros meses del embarazo? Creemos que no se deba usar durante las doce primeras semanas de embarazo mientras no se conozcan estudios y comunicaciones bien documentadas.

ACTINOMICINA $V$. Llama la atención el hecho de que el $50 \%$ de fetos de ratas a las cuales se les ha administrado del 7: al 9० día de embarazo aún a dosis inferiores a las terapéuticas, nacen con severas malformaciones de su sistema óseo, nervioso, cardiovascular, digestivo y ocular. Idénticos resultados se han obtenido en conejas con dosis cuatro veces menores a las terapéuticas. Ello nos obliga a ser muy cautos en su aplicación durante el embarazo (37).

SULFONAMIDAS. Todas franquean la barrera placentaria sin dificultad. La sulfadiazina y el sulfafurazol son las que dan concentraciones más elevadas en el líquido amniótico. Su eliminación se hace con mayor lentitud en el feto que en la madre. Si se inyectan a la madre por vía venosa, se encuentran en concentración equilibrada en la sangre fetal 15 minutos más tarde. La sulfisoxazole (Gantri- 
sin) y las sulfas de acción prolongada (Madribon, Kinex, Sulfametoxipiridazina, etc.) y en general todas las sulfonamidas absorbibles administradas a la madre por largo tiempo y hasta el momento del parto, o a dosis altas durante el trabajo de parto, dan en el recién nacido niveles sanguíneos que persisten hasta 15 días después del parto $y$ pueden causar ictericia y kernícterus aún con niveles de bilirrubina tan bajos como de 12 mgrs. (36).

También las sulfas administradas a la madre pueden dar en el recién nacido un factor de hiposolubilidad sanguínea (38), (97).

HIPOGLICEMIANTES ORALES. Son considerados como derivados del grupo sulfanilamídico. En animales de experimentación se han encontrado efectos teratogénicos en el $36 \%$ de los casos cuando se ha empleado CORBUTAMIDA y en el $2 \%$ cuando se ha administrado TOLBUTAMIDA (39). También se han descrito algunos casos de malformaciones teratológicas y aún de mortalidad atribuíble a estas substancias (40). Sin embargo la acción teratológica de la tolbutamida ha sido puesta en tela de juicio y hay autores que se muestran partidarios de su libre uso durante el embarazo (41). Con todo la prudencia nos aconseja ser muy cautos en el empleo de estos fármacos en mujeres embarazadas, ya que en un estudio comparativo hecho en ratas sobre posible acción teratogénica entre los hipoglicemiantes orales a dosis de 500 mgrs. diarios durante nueve a diez días, la tolbutamida dio $41 \%$ de malformaciones, $37 \%$ la clorpropamide y $90 \%$ la clorbutamida (103)

$Y$ cabe informar que en relación con la INSULINA, ésta atraviesa fácilmente la barrera placentaria y tiene cabal indicación en el tratamiento de la diabetes mellitus de mujeres embarazadas ya que es un substituto de la tolbutamida pero carente de efectos secundarios desfavorables; por su acción benéfica innegable en estos casos, ayuda a la prevención del aborto, de la muerte fetal, del polihidramnios y de las malformaciones fetales que puede acarrear este trastorno metabólico. Pero sí se emplea en mujeres embarazadas para producir choques en tratamientos siquiátricos produce alteraciones condrodistróficas y muerte fetal hasta en el $36 \%$ de los casos (43). También se han reportado manifestaciones nerviosas graves en el recién nacido después de comas insulínicos en la madre. Hay una correlación clara entre las malformaciones fetales y los accidentes vasculares de las madres diabéticas, mas no entre las malformaciones fetales y la insulinoterapia (44).

\section{$\checkmark$ - HORMONAS SEXUALES}

Son éstas las que más han llamado la atención de los investigadores y en su contra se han presentado casos tanto de masculinización como de feminización de los fetos. Recordemos que en el embrión humano las gonadas están presentes desde la 5 a y 6 a semanas pero de manera indiferenciada; ya al final de la sexta semana se desarrolla el sistema masculino o femenino y al final de la octava semana el sexo queda definido. Entre la décima y undécima semanas se han unido definitivamente las varias porciones del riñón. El período crítico de diferenciación está entre la quinta y la octava semana, y en los embriones femeninos la vulva y el vestíbulo están formados hacia la semana 17; al final de la semana 20 ha quedado terminado el desarrollo de los genitales externos (53). 
PROGESTERONA. Usada en el primer trimestre del embarazo no produce alteraciones fetales (50). La progesterona que administramos en la actualidad, privada ya de la acción andrógena de las primeras preparaciones, es de inocuidad comprobada (2). Sin embargo los efectos embriopáticos que pudiesen aparecer se podrían explicar por la acción de metabolitos intermedios, de gran poder andrógeno y no a la progesterona misma. Algunos autores le atribuyen una acción depresiva sobre el centro respiratorio del niño y por lo tanto no recomiendan su uso al final del embarazo (49).

LOS PROGESTOGENOS de síntesis halogenados tipo Clormadinona se pueden utilizar sin riesgo alguno en cualquier edad del embarazo (47). Mas no así los del grupo 19 NOR (derivados de la testosterona y de la nortestosterona), de manera especial el 17 alfa-etinil-testosterona (Norlutin) (51), (52), cuya acción masculinizante sobre productos hembras es muy marcada aun cuando desigual, variando de la hipertrofia aislada del clítoris a una aparente intersexualidad. Estas anomalías tienen relación directa con la dosis y con el tiempo de administración. Las anomalías más avanzadas se encuentran cuando estos progestógenos han sido administrados entre la sexta y la duodécima semana, o sea al final del primer trimestre. Los cambios observados son patentes, pero no son estrictamente teratógenos ya que sólo producen crecimiento excesivo del clítoris y fusión de los labios vulvares, formados ya normalmente (48). Esta alteración es transitoria y pasajera que no requiere tratamiento alguno, salvo el quirúrgico si el falo es muy largo y la fusión de los labios cierra por completo el orificio vaginal.
ESTROGENOS. Los hallazgos en experimentación animal son diametralmente opuestos y diferentes a los observados en la clínica con seres humanos. Bongiovanni (54) y Grumbach (55) han informado de siete casos de seudohermafroditismo en niñas cuyas madres habían recibido dietil-etil-bestrol. Bongiovanny cree que aun cuando los 17 cetosteroides de estas niñas eran normales, los estrógenos estimularon y provocaron una hiperplasia suprarrenal con aumento lógico de factores androgénicos responsables de la virilización. De acuerdo a los estudios de Levitz y colaboradores la placenta no deja pasar prácticamente sino los estrógenos no conjugados (44). En esta forma podemos considerar a los estrógenos, por ahora, como inofensivos para el feto. Bien conocido es el mecanismo de "neutralización" de los estrógenos administrados a embarazadas por parte de placentas funcionalmente normales, lo que se demuestra por la falta de respuesta en la citología funcional vaginal tanto a la administración oral como parenteral de los estrógenos.

OVULOSTATICOS ORALES. Es bastante frecuente la ingestión accidental de estos compuestos al comienzo de un embarazo desconocido, o como prueba de embarazo. Solamente se ha reportado en 1962 un caso de Dunonitz con formaciones urogenitales en un niño cuya madre había ingerido durante tres días una asociación de etisterona-estradiol a la cuarta semana del embarazo (44). Aun cuando estas asociaciones tienen dosis muy débiles de esteroides sexuales y parecen estar carentes de todo riesgo, no las podemos evaluar de manera precisa y debemos atenernos a las conclusiones de la OMS en 1966 (56). 
ANDROGENOS. De uso inexplicable durante la gestación. Se ha demostrado el paso de la testosterona marcada de la madre al feto. Si se administran de la octava a la décimatercera semanas del embarazo producen masculinización de los fetos femeninos, consistente en fusión del labio escrotal sin hipertrofia del clítoris según estudios de Seringe, de Bach y de Loewe-Lyon en 1963 (44). Efectos similares se alcanzan con productos anabólicos. Estos signos de masculinización producidos aún con bajas dosis no tienen tendencia a progresar sino más bien a regresar después del nacimiento.

ESTEROIDES CORTICO SUPRARRENALES. Algunos autores afirman que pueden producir hendidura palatina (57), (58), (59) en proporción que va descle $0,5 \%$ y $2 \%$ hasta $8,5 \%$ respectivamente. Otros autores no han encontrado alteraciones que se les puedan atribuir (60). Se han descrito casos de deficiencia adrenocortical aguda en hijos de madres a quienes se administraron glucocorticoides durante el embarazo (61). Por este motivo todo recién nacido de madre sometida durante la gestación a esta terapia debe ser vigilado cuidadosamente al menos durante un mes. En estos recién nacidos se tiene por norma no aplicar terapia cortisónica cuando el tratamiento materno tenía función substitutiva; en cambio, cuando la producción cortisónica materna era normal y la terapia no era substitutiva, se debe administrar al recién nacido para compensar el aporte exógeno materno interrumpido de manera brusca e intempestiva; se aconsejan 5 mgrs. de cortisona el primer día y luego ir bajando 1 mgr. diario (2). Se recomienda usar los corticosteroides solamente después del primer trimestre del embarazo y únicamente en aque- llos casos en los cuale ssu aplicación se haga imprescindible.

HORMONAS TIROIDEAS. Las hormonas tiroideas atraviesan con dificultad la barrera placentaria, sobre todo al final del embarazo, siendo la triyodotironina la que la traspasa con más facilidad debido a su unión con las proteínas plasmáticas (62), (63). Ello hace que podamos usar en la futura madre el tiroides desecado aún a dosis altas, cuando está indicado, sin riesgo para el producto.

DROGAS ANTITIROIDEAS. EI tiroides fetal empieza a funcionar a partir de la 12: a 14! semanas. El yodo 131 radioactivo, el propil-tio-uracilo, el tapazole y aún el yoduro de potasio pueden producir bocio en el feto (62), (63) el que ordinariamente es transitorio en el recién nacido y no altera la función tiroidea, pero que sí puede acarrear alteraciones respiratorias por compresión de la tráquea, lo que obliga, en algunas oportunidades, a resecar el istmo del tiroides. La yodotironina puede mejorar estos síntomas pero su acción es muy lenta (50). Con alguna frecuencia se presentan luego crisis tiroideas semejantes a la tirotoxicosis, las que se tratan con lugol o con yoduros a dosis gradualmente decrecientes.

A veces el yodo 131 radioactivo (65) y el propil-tio-uracilo (63) producen involución del tiroides fetal y aún hipotiroidismo.

En la experimentación animal se ha encontrado bocio coloidal como también adenomas cromófobos hipofisiarios. Recordemos que el tiroides fetal tiene una avidez por el yodo 131 radioactivo diez veces mayor que el de la mujer adulta; por este motivo no se debe emplear jamás en la mujer embarazada ni aún en el período de lactancia (3). 
Debemos tener sumo cuidado con el empleo de algunos medios de contraste muy usados en radiología cuya materia básica es el yodo; algunos de ellos como el Teridax dan niveles muy altos de yodo, el que se une íntimamente a las proteínas a punto tal que se ha podido detectar varios años más tarde (11).

Autores como Adams sostienen que ante una tirotoxicosis las drogas antitiroideas no tienen efecto nocivo alguno sobre el feto siempre y cuando que su indicación sea perfecta y que se administre medicación bloqueadora hipofisiaria para prevenir el bocio exoftálmico del recién nacido (66).

\section{VI - VITAMINAS}

B-6. Algunos autores atribuyen al exceso de ingestión de B-6 o piridoxina por la madre al final del embarazo, un síndrome convulsivo del recién nacido (67).

VITAMINA D. La sobredosificación de Vitamina $D$ produciría calcificaciones a nivel de la placenta, hipercalcificación de los huesos del cráneo causante de distocia, hipercalcemia y calcificación de las articulaciones y de los tejidos blandos de manera especial de los riñones y gruesos vasos (30). Lo que ha sido descrito como retención temprana de calcio seguida de calcificación del esqueleto puede representar una transferencia del mineral a lugares anormales. UItimamente ha sido descrito un síndrome relacionado con ingestión excesiva e innecesaria de Vitamina D: estenosis aórtica supravalvular, deficiencia mental, hipercalcemia idiopática; a los Rayos X se aprecia osteoesclerosis de la base del cráneo (104).

VITAMINA K. Suministrada a dosis exageradas (72 mgrs. o más) du- rante el trabajo de parto, aumenta la bilirrubinemia fetal y puede dar hemólisis en el recién nacido (69). Esta agresión se puede explicar bien por acción tóxica sobre la célula hepática, bien por una competición enzimática que disminuiría la conjugación de la bilirrubina o bien por un aumento de la hemólisis de los glóbulos rojos producida por inestabilidad transitoria del glutatión reducido. Es indispensable proporcionar al pediatra el dato exacto de la Vitamina $K$ que se ha administrado a la madre para el correcto uso que éste necesite hacer de ella, al ser requerida por el recién nacido. La dosis máxima que podemos usar en la madre no debe nunca sobrepasar de los 10 mgrs. en total.

\section{VII - ANTICOAGULANTES}

HEPARINA. Por su elevado peso molecular atraviesa muy difícilmente la barrera placentaria y sus efectos son de duración limitada; no se conocen accidentes atribuíbles a ella; además el sulfato de protamina es un antiheparínico de acción constante y rápida. Por este motivo se le considera como la droga de elección para el tratamiento de lesiones tromboembólicas de las embarazadas. Pero es aconsejable un riguroso control del tiempo de protrombina cuando se emplee (2).

DICUMARINA y FENILINDANDIONA (Dicumarol y Coumadin). Pueden producir hemorragias intracraneanas en el feto aún en casos en que el tiempo de protrombina materno no esté en los límites considerados como mínimos pero no peligrosos (70) (71). No se deben usar en la mujer embarazada (101).

\section{VIII - TIAZIDAS}

Recientes publicaciones inglesas han reportado numerosos casos de 
trombocitopenia neonatal, mortal en muchos de ellos, en hijos de madres a quienes se administraron tiazidas por tiempo prolongado aún a bajas dosis, o a dosis altas al final del embarazo (105). El efecto teratógeno de las tiazidas no se ha valorado de manera completa pero se considera pequeño (106). Sin embargo se han descrito defectos de las extremidades de fetos de ratas a las que se les había suministrado dosis altas de acetazolamida (107). Por su fácil paso transplacentario com probado (114) producen las mismas alteraciones electrolíticas tanto en la madre como en el feto (115); y recordemos que ya se han descrito muertes maternas atribuíbles exclusivamente al uso de tiazidas (116). Debemos también tener presente la acción diabetógena de las tiazidas en la mujer, la que puede tener efecto retardado en el producto; y esta reacción de hiperglicemia materna, si es sostenida, puede dar fetos de alto peso con hipertrofia de los islotes pancreáticos y peligro de muerte fetal y neanatal (108) (110).

Las tiazidas son diuréticos potentes y útiles pero pueden ser peligrosos para la madre y para el feto (64).

Algunos diuréticos como el Cloruro de amonio, al igual que las tiacidas, pueden producir en el feto los mismos trastornos electrolíticos observados en la madre cuando se administran de manera poco vigilada (109), (111).

\section{IX - MEDICAMENTOS CITOSTATICOS}

Usados durante el embarazo para provocar el aborto terapéutico y en el tratamiento de la leucemia, de la enfermedad de Hodgkin y en las neoplasias (73), (74), (75), (76) tales como el Aminopterin, el Purinetol, el Busulfan, el Trietilen-tio-fosforamida
(Thiotepa), las mostazas nitrogenadas, la Aminopterina (Methotrexate), la 6 mercato purina, el Clorambusil, etc. Todos ellos tienen efectos abortivos y teratogénicos (77), (79), (80), (81). Las malformaciones se pueden producir en cualquiera de los órganos de la economía y dependen de la edad del embarazo en que se administren lo mismo que de las dosis y duración del tratamiento. Administrados al final del embarazo producen menos malformaciones pero pueden llevar al parto prematuro (72). El período de mayor peligro para el feto es la administración en el primer trimestre. El tratamiento hasta donde sea posible, debe posponerse para el segundo o tercer trimestre y se usarán las dosis mínimas eficaces en el caso de que se considere que la quimioterapia sea indispensable (78).

\section{$X$ - BIBLIOGRAFIA}

1 GONZALEZ-RAMOS, M. Curso internacional de Genética Médica dictado en Lima. Sept. 22 a 26, 1968.

2 CASTELAZO-AYALA, L. y DOMINGUEZ-ROBLES, H. Factores físicos y farmacológicos que afectan al feto. Ginec. Obstet. Méx. 20 : 425, 1965.

3 CHASSAGNE, P. Risques thérapeltiques chez la femme en ceinte. La Rev. du Prat. 7: 12, 1966.

4 CHASSAGNE, P. Précautions thérapeutiques chez la femme en ceinte. Press. Méd. Paris. $71: 1075,1963$.

5 USANDIZAGA, J. A. Debemos vacunar contra la poliomielitis a las embarazadas? Medicamenta. 374: 341, 1960.

6 COBRINIK, R. W. The effect of maternal narcotic addiction on the newborn. Review of literature and report of 22 cases. Pediatrics. 24: 228, 1959.

7 GOODFRIEND, M. J.; SHEY, I. A. and KLEIN, M. D. The effects of maternal narcotic addiction on the newborn. Am. J. Obst. and Gynec. 71: 29. 1956.

8 APGAR, V. and BRODIE, B. B. Am. J. Obst. Gynec. 64: 1368, 1952. 
9 ECKENHOFF, J. E.; HOFFMAN, G. L. and FUNDENBURG, L. W. N-allylnormorphine: an antagonist to neonatal narcosis producet by sedation of the parturient. Am. J. Obst. and Gynec. 65: 1269, 1953.

10 FRIEND, D. G. Farmacodinamia en el feto. Clin. Pharmacol. Ther. 4: 141, 1963.

11 DOREY, H. H. Médicaments donnés durant la gestation et aprés la naissance. Effets néfastes por le foetuc et le nouveau-né. L'Union Méd. du Canada. 93: 555, 1964.

12 VAN S. MAECK, J. and STRAUSFELD, K. Analgesia y anestesia en el parto prematuro. Clin. Obst. y Gin. 707, 1964.

13 GILLESPIE, L. Tabaquismo y peso bajo del recién nacido. Clin. Obst. y Gin. 658, 1964.

14 CORONEL, J. G. ; BUSTAMANTE, G. y URIBE-BOTERO, G. Prematuridad en nuestro medio. Rev. Col. Pediatría. 10: 275, 1968

15 ZABRISKIC, J. R. Effect of cigaret smoking during pregnancy. Obst. and Gynec. 21: 405,1963

16 O'LANE, J. M. Some effects of maternal cigaret smoking. Obst. and Gynec. 22: 181, 1963.

17 SIMPSON, W. J. Preliminary report of cigarrette smoking and the incidence of prematurity. Am. J. Obst. and Gynec. 73: 808, 1957.

18 BUDNICK, I.; LEIKIN, S. and HOECK, L. Effects on the newborn infant of the Reserpine administered ante partum. J.A.M.A. 90: 286, 1955

19 BOGGS, T. R. Jr.; LUCINE, A. A. and NEEDLEMAN, H. L. Promethazine hydrochlorhide and neonatal bilirubin concentration. Jr. Pediatric. 67: 160, 1963

20 SOBEL, D. Fetal damage due to E. C. T. insulin coma, clorpromazine or reserpine. Am. Arch. Gen. Psychiat. 2: 606, 1960.

21 ALTIRIBA, J. E. y col. Causas yatrógenas del sufrimiento fetal. En el libro Sufrimiento Fetal en el parto. Ed. Balmes. 197, 1967.

22 CORNER, B. D. Congenital malformations. Méd. J. Southwest. 77: 46, 1962.

23 COHEN, Y. Le passage transplacentaire des médicaments. Thérapie. 16: 509. 1961.

24 VAN CLEVELD, S. Coagulation disorders in the newborn period. Jr. of Pediat. 54: 633, 1959.
25 WARKANY, J. and FRASER, F. C. Prenatal factors in diseases of children. En Nelson, W. E. Textbook of pediatrics. 7 ed. W. B. Saunders Co. edit. Philadelphia, pág. 242, 1959.

26 MAUER, F. M.; DE VAUX and WAND LAHEY, M. E. Neonatal and maternal thrombocytopenic purpura due to quinine. Pediatrics. 19: 84, 1957.

27 The Médical Letter. Vol. 9 № 222, 1967.

28 SOLCISKI, A. y col. Malformación fetal y quinina. Instantanes Médicaux. 4: 297, 1966.

29 CHARLES, D. J. Obst. and Gynec. Britl. Emp. 61: 750, 1954.

30 CHASSAGNE $P$, et GEORGES-JANET, L. Influence sur le fétus de certains traitements appliqués á la femme enceinte. Ann. Pediat. 37 : 365, 1961.

31 SANCHEZ-CARVAJAL, A. M. Efectos adversos de las drogas y otros agentes sobre el feto. Rev. Obst. Gin. Venezuela. 28: 197, 1968.

32 KLINC, A. H.; BLATTNER, R. J. and LUNIN, M. Efecto transplacentario de las tetraciclinas sobre los dientes. J.A.M.A. 188: 178, 1964.

33 COHLAN, S. Q.; BEVELANDER, G. and TIAMSIG, T. Growth inhibition of prematures receiving tetracycline. Am. J. Dis. Child. 105: 453, 1963.

34 WALLMAN, I. S. and HILTON, H. B. Teeth pigmented by tetracycline. Lancet. 1: 827 , 1962.

35 SURHERLAND, J. M. and KELLER, W. H. Novobiocin and neonatal hyperbilirrubinemia investigation of relationship in epidemic of neonatal hyperbilirrubinemia. Am. J. Dis. of Child. 101: 447, 1961

36 SILVERMAN, W. A.; ANDERSON, D. H.; BLANC, W. A. and CROZIER, D. N. Difference in mortality rates and incidence of kernicterus among premature infants allotted to 2 prophylactic antibacterial programs. Pediat. 18: 614, 1956.

37 TUCKMANN-DUPLESIES, $H$. and MERCIERPAROTL. The teratogenic action of the antibiotic actinomycin din congenital. Ed. G E. W. Wolsten Holme and C. M. O'Connor, Boston, Little Brown, pág. 115, 1960.

38 VAN CREVELD, S. Coagulation disorders in the newborn period. Jr. of Pediat. 54: 633, 1959. 
39 SUGAR, S. J. Use of the sulfonylureas in diabetes mellitus. Ann. New York Acad. Sc. $71: 256,1957$.

40 LARSONY and STERKY, C. Possible teratogenic effect of tolbutamide in a pregnant prediabetic. The Lancet. 2: 1424, 1960.

41 GHANEM, M. H. The management of pregnant diabetes with sylponyl-urea compounds. Alexandria M. J. 6: 14, 1960.

42 The Médical Letter. No 238, 1968.

43 WICKES, I. G. Foetal defects following insulin coma therapy in early pregnancy. Brit. J. 2: 1029, 1954 .

44 LAPLANE, R. Y GEORGES-JANET. EI riesgo fetal en los tratamientos endocrinos. La Presse Médical. 76: 785, 1968.

45 MELLIN, G. W. y KATZENSTEIN, M. Meclizine and fetal abnormalities. Lancet. 1 : 222, 1963.

46 YERUSHALMY, J. and MILKOVICH, L. Evaluation of the teratogenic effect of meclizine in man. Amer. J. Obstet. Gynec. 93: 553, 1965.

47 DUARTE-CONTRERAS, A. y ZUÑIGA, R. Progestógenos. Rev. Col. Obst. Gin. 14: $569,1963$.

48 APGAR, V. Problema de los fármacos en la gestación. Clin. Obstet. y Ginec. 623, 1966.

49 SMITH, R. Control de la respiración. En Anestesia para niños. Ed. Morvi Com. New York 48, 1959.

50 DUCHARME, J. R. Endocrine problems of the newborn infant. Ped. Clinics. N. A. 8: 565,1961

51 WILKINS, L. Masculinization of female fetus due to use of orally given progestins. J.A.M.A. 172: 1028, 1960.

52 WILKINS, L. Masculinization of the female foetus associated with the administration of oral and intramuscular progestins during gestation: non adrenal pseudohermafroditism. J. Clin. Endocr. 18: 559, 1958.

53 LANGMAN, J. Medical Embriology. Williams and Wilkins, Baltimore, 1963.

54 BONGIOVANNI, A. M.; DI GEORGE, A. M. and GRUMBACH, M. M. Masculinization of the famale infant associated with estrogenic therapy alone during gestation: four cases. J. Clin. Endocrinol and Metab. 19: 1004, 1959.
55 GRUMBACH, M. M. and col. J. Clin. Endocrin. 19: 1369, 1959.

56 Aspectos clínicos del empleo de gestágenos orales. Resumen del informe No 326 de la OMS. Rev. Col. Obst. Gin. 18: 353, 1968.

57 BONGIOVANNI, A. M. and MCPODDEN, A. J. Steroids during pregnancy and possible fetal consequences. Fertility and Sterility. $11: 181,1960$.

5858 HARRIS, J. W. S. and ROSS, I. P. Cor. tisone therapy in early pregnancy. Lancet. 1: $1045,1956$.

59 DOIG, R. K. and COLTMAN, O. M. Cleft palate following cortisone therapy in early pregnancy. Lancet 2: 730, 1956

60 LEYSSAC, P. P. Suprarrenal steroids and pregnancy. Ugeskrift Laeger. 121: 33, 1959.

61 HCTTINGER, A. Insuffisance cortico-surrénalienne chez le nouveau-né de mére traité par la cortisone. Ann. Pediat. 37: 2469, 1961.

62 RAY, E. W.; STERLING, K. and GARDNER, L. I. Congenital cretinism associated with I 131 therapy of the mother. (abstract). A.M.A. Jr. Dis. Child. 90: 506, 1959.

63 BONGIOVANNY, A. M.; EBERLEIN, W. R.; THOMAS, P. Z. and ANDERSON, W. B. Sporaid goiter of the newborn. J. Clin. Endocrinol and Metab. 16: 146, 1956.

64 RODRIGUEZ DE LA FUENTE, F.; CARDENAS-VARELA, S. ; AREVALO-RODRIGUEZ, J. ; MORA-AREVALO, S. Y NASSAR-CONTRERAS, $M$. Consideraciones hemodinámicas en el manejo analgésico y anestésico de la embarazada en trabajo de parto con diagnóstico de toxemia. Gin. Obstet. Méx. 153: 1, 1969.

65 FISHER, W. D. ; WOORHESS, M. L. and GARDNER, L. I. Congenital Hypothiroidism in infant following maternal I 131 Therapy (Clinical pathological conference). Jr. Pediatr. 62: 132, 1963.

66 ADAMS, A. E. The effect of antithiroid drug. Anat. Rec. 104: 421, 1949.

67 JULIEN-MARIE, A.; HENNEQUET, A. ; LYON, C.; DEBRIS, P. et LE BELLE, J. C. Dépendence á la pyridoxine: une maladie métabolique. Rev. Neurol. 105: 406, 1961.

68 Comunicación personal de Laboratorios Glaxo, el 10 de septiembre de 1969.

69 LUCEY, J. F. and DOLAN, R. G. Hyperbilirrubinemia of new horn infants associated 
with the parenteral administration of a vitamin $K$ analogue to the mothers. Pediatrics 23: 553, 1959.

70 GORDON, R. R. and DEAN, T. Fetal deaths from antenatal anticoagulant therapy. Brit. M. J. 2: 719, 1955.

71 JOSEPH, R. Fetal haemorrhages induced by the administration of anticoagulants to the pregnant woman. Sem. Ther. 38: 115, 1962.

72 SOKAL, J. E. and LESSMAN, E. M. Effect of cancer chemotherapeutic agents on the human fetus. J.A.M.A. 172: 1765, 1960.

73 MELTZER, H. J. Congenital anomalies due to attempted abortion with 4-aminopteroglutamic acid. J. Amer. Med. Ass. 161: 1253, 1956.

74 SMITH, R. B. W., SHEEHY, T. W. and ROTHBERG, $H$. Hodgkings disease and pregnancy. Arch. Inst. Med. 102: 777, 1958.

75 SBOTTON, D. and MONIE, I. W.: Possible teratogenic effect of chlorambucil of a human foetus. J.A.M.A. 186: 174, 1963.

76 THIERSCH, J. B.: Therapeutic abortion with a folic acid antagonist administered by the oral route. Am. J. Obstet, Gynec. 63: $1298,1952$.

77 REVOL, L., VIALA, J., PELET, J. and CROIZAT, P. Hodgkin's disease, linphosarcoma, reticulosarcoma and pregnancy. New Rev. Franc. Hémat. 2: 311, 1962.

78 STUTZMAN, L. y SOKAL, J. E. Uso de anticancerosos durante la gestación. Clínicas Obstét. y Ginecol. 416, 1968.

79 GREENBERG, H. L. and TANAK, K. R. Congenital anomalies probably induced by ciclophosphamide. J. Amer. Med. Ass. 188: 423, 1964.

80 LUTWAK-MANN, C. and HAY, M. F. Effect on the early embryc of agents administered to the mother. Brit. Med. J. 2: 944, 1962.

81 KARNOFSKY, D. A. Late effects of inmunesupressive anticancer drugs. Fed. Proc. 26: 925, 1967.

82 GREENBERG, M. and col. Effect of Smalpox vaccination during pregnancy on incidence of congenital malformation. Pediatrics. 3 : 456, 1949.

83 MCARTHUR, P. Congenital vaccinic and vaccinic gravidarum. Lancet. 2: 1104, 1952.

84 BURNETT, S. G. Monphinism in pregnancy and the newborn. The Med. Herald 27 : 161, 1920.
85 HAGERMANN, D. D. and VILEE, G. A. Transport functions of the placenta. Phys. Reviews 40: 313, 1960.

86 USUBIAGA, J. E. Mesa Redonda sobre Analgesia y Anestesia obstétrica en el IX Congreso Colombiano de Anestesiología. Medellín, agosto 13-16, 1969.

87 EARLE, R. Congenital salicylate intoxication. Report of a case. New Eng. J. Med. 265: 1003, 1961.

88 SNYDER, F. F. Obstetric analgesia and anesthesia. W. B. Saunders Comp. Philadelphia, 1949.

89 COSMI, E. and MORISHIMA, H, O. Actas Symposium. "Problems of foetal distress. Siene (Italia), sept. 1966.

90 BOTELLA-LLUSIA, J. y col. Acta Gin. 12: $661,1961$.

91 DEXEUS, J. M. y GONZALEZ-RUIZ, J. Progres. Obstet. Gin. 6: 11, 1963.

92 DESMONIL, M. M. Phisical signs accompning transition. In Phisical Diagnosis of the newborn. Aorty sixth Ross Conference on Pediatric research, Columbus, Ohio, Ross Laboratories, 18, 1964.

93 YOUNG, I. M. Placental transfer of hexametonium bromide in rabit and its apperance in amniotic fluid. J. Physiol. 166: $4,1956$.

94 HALLUM, J. and HATCHUEL, W. Congenital paralytic ileus in a premature baby as a complication of hexamethonium bromide therapy for toxemia of pregnancy. Arch. Dis. Child. 29: 354, 1954.

95 KING, C. T. G. Teratogenic effects of meclizine hydrochlorhide on the rat. Science 14: 353, 1963.

96 LENZ, W. Malformations caused by drugs in pregnancy. Amer. J. Dis. Child. 112: 99, 1966.

97 BROWN, A. K. and CEVIK, N. Hemolysis and jaundice in the newborn folowing maternal treatment with sulphamethoxypiridazine. Pediatrics. 36: 742, 1965.

98 SANCHEZ CONTRERAS, J., KASSEM CAMACHO, J. Y MARADIEGUE MENDEZ, E. La toxoplasmosis como factor de infertilidad. Ginec. Obste. Méx. 151: 539, 1969.

99 SANCHEZ CONTRERAS, J. Y RUIZ VELASCO. Algunos factores etiológicos del aborto habitual. Obste. Ginec. Méx. 21: 677. 1966. 
100 SPNIGAR, L. I. y EDELMAN, M. H. Parasitosis y embarazo. En Complicaciones médicas, quirúrgicas y ginecológicas en el embarazo. De Rovinsky, J. J. Ed. Interamericana S. A. 2a. Ed. México. 603, 1964.

101 BOCQUET, L. Les dangers des anticoagulants au cours de la grossesse. Thérapie. 69: $507,1961$.

102 WEISS, C. F., GLAZKO, A. J. and WES TON, J. K. Chloranphenicol in the new born infant. New England J. Med. 262 $787,1960$.

103 MEYER, R. Teratogenic effects of sulfonylura derivatives 2-deoxy glucosa and sodium fluro-acetate in rats. Fourth Congress of the International Diabetes Federation, Geneva, Switzerland, july 10-14. 401, 1961.

104 GARCIA, R. E., FIEDMAN, W. F., KABACHA, M. M. and ROWE, R. A. Idiopatic hypercalcemia and supravalvular aortic stenosis. Documentation of a new Syndrome. New England J. Méd. 271: 117, 1964.

105 RODRIGUEZ, S. U., LEIKIN, S. L. and HILLER, M. C. Neonatal thrombocytopenia associated with ante-partum administration of thiazide drugs. New England J. Med. 270: $881,1964$.

106 GRAY, M. J. Uso y abuso de las tiazidas en la gestación. Clínicas Obstét. y Ginecol. 568, 1968.

107 LAYTON, W. M. and HALLESY, D. W. Deformity of forlimb in rats: association with high doses of acetazolamide. Science 149: 306, 1965.

108 ESBANSHADE, J. $H$. Jr. and SMITH, R. J. Thiazides and pregnancy: a study of carbohydrate tolerance. Amer. J. Obstet. Gynec. 92: 270, 1965.

109 GOODLIN, R. C. and KAISER, I. The effect of amonium chloride induced maternal acidosis on the human fetus at term.
Ann. J. M. J. C. 233: 662, 1957, and 235: 555,1958

110 MINKOWITZ, S., SOLOWAY, H. B., HALL, J. E. and YERMAKOV, V. Fatal hemorrhagic pancreatitis following chlorotiazide administration in pregnancy. Obstet. and $\mathrm{Gy}$ nec. 24: 337, 1964.

111 KRAUS, C. W., MARCHESE, J. R. and YEN, S. S. C. Prophylactic use of hydrochloro thiazide in pregnancy. J.A.M.A. 198: 1150,1966

112 SCHNIDER, S. M. Foetal and neonatal effects of drugs in obstetrics. Anesth. Analg. 45: 372, 1966.

113 APGAR, V. Perinatal sequelae of drugs and anesthetic agents. Harper Hostp. Bull. 24: 135,1966

114 GARNET, J. D. Placental transfer of chlorothiazide. Obstet. and Gynec. 21 : 123,1963

115 BATTAGLIA, F., PRYSTOWSKY, H., SMISSON, C., HELLEGERS, $A$. and BRUNS, $P$. Fetal blood studies. XIII. The effect of the administration of fluids intravenously to mothers upon the concentrations of water and electrolytes in plasma of human fetuses. Pediatrics. 25: 2, 1960.

116 SCHIFRIN, B. S., SPELLACY, W. N. and LITTLE, W. A. Maternal death associated with excesive ingestion of a chlorothiazide diuretic. Obstet. and Gynec. 34: 215 1969

117 BCLLETTI, M. e CROATTO, L. Sulla surditá da passagio transplacentare di streptomicina. Acta Pediat. Lat. Reggio. 11: 1, 1958.

118 SWALLOW, J. N. Discolvoration of primary dentition after maternal tetracycline ingestion during pregnancy. Lancet. 2: 611, 1964.

119 RENDLE-SHORT, T. J. Tetracycline in teeth and bones. Lancet 1: 1188, 1962. 